\title{
Anisotropies of the highest energy cosmic-ray events recorded by the Pierre Auger Observatory in 15 years of operation
}

\author{
Lorenzo Caccianiga ${ }^{* a}$ for the Pierre Auger Collaboration ${ }^{b \dagger}$ \\ ${ }^{a}$ Istituto Nazionale di Fisica Nucleare, Sezione di Milano, Italy \\ ${ }^{b}$ Observatorio Pierre Auger, Av. San Martín Norte 304, 5613 Malargüe, Argentina \\ E-mail: auger_spokespersons@fnal.gov \\ Full author list: http://www.auger.org/archive/authors_icrc_2019.html
}

\begin{abstract}
To understand the origin of ultra-high energy cosmic rays (UHECRs), the study of the distribution of their arrival directions has always been of capital importance, despite the difficulties that arise from the deflection they suffer due to magnetic fields. In particular, the highest-energy region, above a few tens of EeV, which extends beyond the observed flux suppression, is of particular interest because of the high rigidities and the suppression of distant sources. We present here the latest results of searches for anisotropies in the arrival directions of the UHECRs detected by the Pierre Auger Observatory. The dataset used for this work is the largest ever recorded from a single observatory at these energies: with nearly 15 years of data, it covers the sky up to a declination of $45^{\circ}$, with an exposure now exceeding $100,000 \mathrm{~km}^{2}$ sr yr. The study of largescale anisotropy indicates an extragalactic origin for the sources of UHECRs with energies larger than $8 \mathrm{EeV}$. A search for anisotropies at small to intermediate angular-scales is presented, which enables a model-independent study of flux patterns at the highest energies. We also study the arrival directions of UHECRs against the distribution of nearby extragalactic matter traced by different catalogs of candidate sources. We discuss the most striking features characterized by the Pierre Auger Observatory, which include the most significant indication of anisotropy in the arrival directions of the highest energy cosmic rays.
\end{abstract}

36th International Cosmic Ray Conference - ICRC2019

24 July - 1 August, 2019

Madison, Wisconsin, USA

\footnotetext{
${ }^{*}$ Speaker.

${ }^{\dagger}$ for collaboration list see PoS(ICRC2019)1177
} 


\section{Introduction}

The quest for the sources of ultra-high energy cosmic rays (UHECRs) is still one of the most important purposes of current high-energy astrophysics. Although recent results on large scale anisotropies have strongly favored an extragalactic origin of the highest energy cosmic rays [1], the specific sources remain unidentified. To pursue this quest, searching for anisotropies and patterns in the arrival directions of the highest energy particles ever recorded is probably one of the most natural paths. Particles above the observed flux suppression [2] at $\sim 40 \mathrm{EeV}^{1}$ are supposed to come only from the local universe, within few hundreds of Mpc, because of the interaction they should undergo with the cosmic backgrounds during their propagation. Given that within this horizon the distribution of matter is inhomogeneous, it is worth searching for anisotropies in the arrival direction distribution of ultra-high energy cosmic rays even if their paths are distorted by the Galactic and extragalactic magnetic fields that they encounter in a (so far) unpredictable way.

\section{The Pierre Auger Observatory}

The Pierre Auger Observatory [3] is the largest detector for UHECRs. It is located in Argentina at a latitude of $\sim-35^{\circ}$, and covers approximately $3000 \mathrm{~km}^{2}$. It is a hybrid detector, using both a surface detector array (SD) composed of 1660 water-Cherenkov detectors and an atmospheric fluorescence detector (FD) made of 27 fluorescence telescopes to observe extensive air showers induced in the atmosphere by primary particles with energies $E>0.1 \mathrm{EeV}$. The Pierre Auger Observatory is taking data since January 2004. Cosmic ray events are reliably reconstructed up to a zenith angle of $80^{\circ}$, which translates into a field of view ranging from $-90^{\circ}$ to $+45^{\circ}$ in declination. The systematic uncertainty on the energy scale is $14 \%$, and the statistical uncertainty in the energy is smaller than $12 \%$ for the highest energy events used in this work [4], while the angular uncertainty is less than $0.9^{\circ}$ at these energies.

\section{The dataset}

For the analyses presented in this work, we used data collected by the Surface Detector of the Pierre Auger Observatory from the $1^{\text {st }}$ of January, 2004 to the $31^{\text {st }}$ of August, 2018. We analyse UHECRs with energy above $32 \mathrm{EeV}$, which corresponds to the highest energy bin analyzed in large scale anisotropy searches, also presented in these proceedings [5]. Events arriving with a zenith angle below $60^{\circ}$ are dubbed as "vertical" while events with zenith angle between $60^{\circ}$ and $80^{\circ}$ are "inclined". Vertical and inclined events undergo different types of selection and reconstruction, that are described in detail in the contribution to this conference reporting on spectrum [2]. For this work, we apply the same relaxed selection used in previous analyses [6]: for vertical events we require that the station with the largest signal is surrounded by at least 4 active stations and that the reconstructed core of the shower falls within an isosceles triangle of active stations, while for inclined events we require only that the station with the largest signal is surrounded by at least 5 active stations. This is a more relaxed selection than the one used in other works presented in these proceedings, such as the analysis of large scale anisotropies, since it is applicable only to the highest

\footnotetext{
${ }^{1} 1 \mathrm{EeV}=10^{18} \mathrm{eV}$.
} 


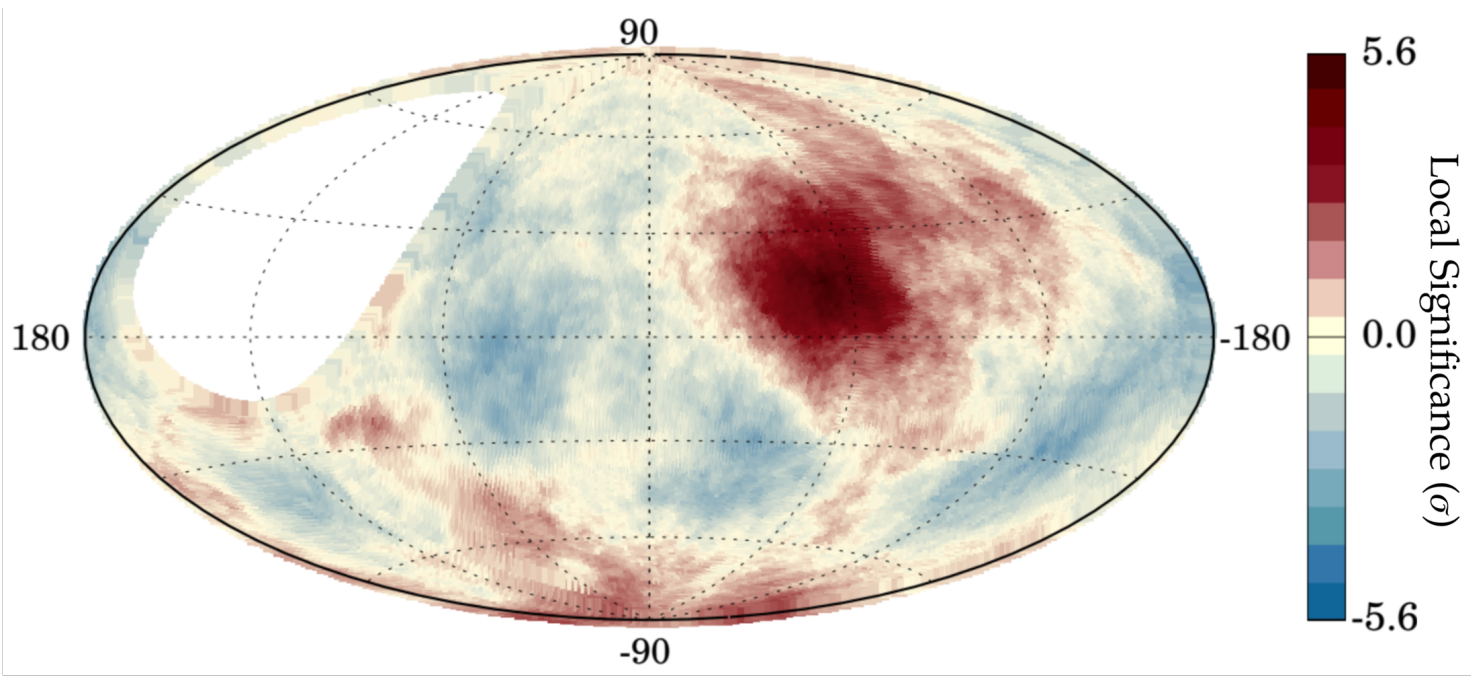

Figure 1: Map in Galactic coordinates of the local significance found when searching for excesses in circular windows with $27^{\circ}$ radius above $38 \mathrm{EeV}$. The post-trial p-value for the most significant excess is $2.5 \%$. See text for details.

energy events that have large footprints on the array. By applying these cuts, we select the highest possible number of events while guaranteeing an accurate reconstruction and exposure estimation. The total number of events selected this way is 2157 , with an exposure of $101,400 \mathrm{~km}^{2} \mathrm{sr} \mathrm{yr}$.

\section{Analyses}

\subsection{Search for overdensities}

The first analysis performed is a model-independent blind search for overdensities over the whole field of view. The search for overdensities was performed with the same methodology used in [6]. The method applied looks for excesses with respect to isotropic expectation in circular regions centered on a $1^{\circ} \times 1^{\circ}$ grid covering the whole field of view. The radius of the circular regions, $\psi$ was varied from $1^{\circ}$ to $30^{\circ}$ in $1^{\circ}$ steps. Also the energy threshold of the events was varied from $32 \mathrm{EeV}$ to $80 \mathrm{EeV}$ in $1 \mathrm{EeV}$ steps. The Li-Ma significance was computed for each excess (or deficit) and then penalized for the scanning trials. This penalization was computed by generating simulated isotropic sets of the same size as the real one and counting how many of them showed an excess with a significance equal or larger than the largest found in our data. The most significant excess is found for $E>38 \mathrm{EeV}$ at equatorial coordinates R.A. $=202^{\circ}, \delta=-45^{\circ}$ in a $27^{\circ}$ radius. In that window, we observe 188 events while we expect 125 from an isotropic distribution of cosmic rays. The local Li-Ma significance is $5.6 \sigma$. When performing the same analysis with random isotropic samples $2.5 \%$ gave an excess of equal or higher significance than the one found. The map of the local significance for $E>38 \mathrm{EeV}$ in $27^{\circ}$-radius windows over the whole sky is shown in figure 1.

\subsection{Correlation with the direction of Cen $\mathrm{A}$}

Centaurus $\mathrm{A}$ is the nearest radio-loud active galaxy, at a distance of less than $4 \mathrm{Mpc}$. The 

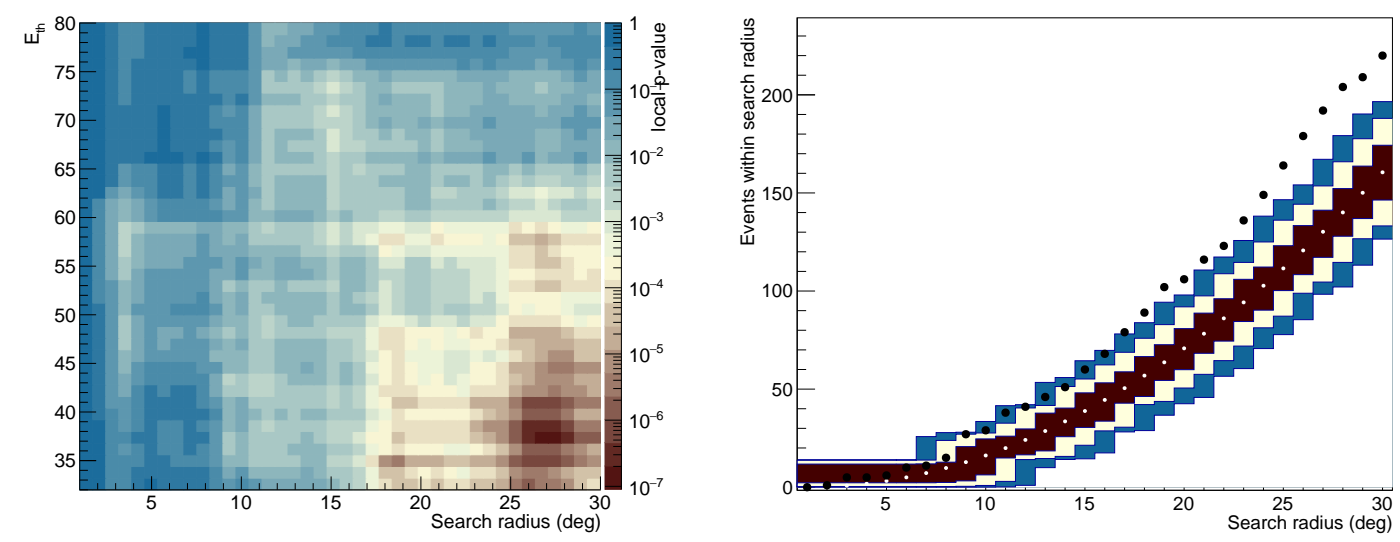

Figure 2: Left: local p-value when searching for excesses around the position of Centaurus A, as a function of search radius and energy threshold. Right: the comparison of number of events with $E>37 \mathrm{EeV}$ within a certain angle from Cen A (black dots) compared to the expectation from a isotropic distribution of UHECRs (white dots) and its 1,2 and 3- $\sigma$ dispersion (red, cream and blue respectively).

search for excesses around its direction was performed by counting the number of events within a certain search radius $\psi$ from the AGN position. The number $n_{\text {obs }}$ thus obtained is then compared to the one expected given an isotropic distribution of UHECRs, $n_{\text {exp }}$. The binomial probability $P$ of obtaining $n_{o b s}$ or more correlating events by chance from an isotropic distribution is computed and considered as local p-values. These values are then penalized as described in the search for overdensities to take into account the trials arising from the scan in energy and in angle. The most significant excess found in the blind search lies $\sim 2^{\circ}$ away from the position of Cen A. It is not surprising then that when scanning in $E_{\text {th }}$ and $\psi$ to find the parameters that give the highest significance, the results are nearly the same as found before: $E_{\mathrm{th}}=37 \mathrm{EeV}$ and $\psi=28^{\circ}$. For these parameters, 203 events were observed while 141 were expected, and the local significance is $5.1 \sigma$ which, when penalized for the scan in energy and angle, leads to a one-sided post-trial significance of $3.9 \sigma$. This is an increase of significance with respect to the results presented at the 2017 edition of the ICRC [7], where it was 3.1 $\sigma .^{2}$ The local p-values in the $E_{\mathrm{th}}-\psi$ space are shown in figure 2, left. In the right panel of the same figure, it is possible to see the comparison of the number of events within a certain radius from Cen A observed for $E>37 \mathrm{EeV}$ to the expectation from an isotropic distribution of cosmic rays (with its 1,2 and $3-\sigma$ dispersion).

\subsection{Likelihood analysis with catalogs of candidate sources}

We also update the search for anisotropies published in [8]. This analysis was motivated by the expectation that brighter objects contribute more to the flux, and the likelihood method allowed us to take into account this hypothesis together with the suppression that is expected to increase with distance because of the interactions of UHECRs along their path. In particular, in this method, a probability map of the arrival distribution of cosmic rays is obtained for each model of candidate sources, and compared to the observed distribution of events using an appropriate test statistics

\footnotetext{
${ }^{2}$ Note that in that case the energy scan ranged from 40 to $80 \mathrm{EeV}$.
} 
(TS). The probability maps are smoothed density maps, obtained by modeling the contribution of each individual object with a Fisher-Von Mises distribution with a certain angular width $\theta$. This smearing angle is one of the free parameters of this analysis, and takes into account the unknown deflections of the UHECRs due to magnetic fields. Each object is weighted based on its relative flux, measured in an appropriate band different for each catalog, and on its distance. The attenuation is computed based on the composition scenario at sources that best reproduces the average composition and spectrum measured at the Auger Observatory (scenario A). An isotropic fraction of events was added to the probability maps obtained this way in order to model the diffuse component of UHECRs due to highly deflected events and faint sources not included in the catalogs. The weight of the anisotropic fraction with respect to the isotropic one, $f_{\text {aniso }}$, is the second free parameter for this analysis. The comparison between the model predictions and the observed data is done through a likelihood ratio test. The likelihood function $(\mathscr{L})$ is the product over the events of the probability map. The likelihood of the null hypothesis (isotropy) $\mathscr{L}_{0}$ is obtained as the product over the events of the directional exposure function in the arrival directions. The test statistics used in this analysis is then $T S=2 \ln \mathscr{L} / \mathscr{L}_{0}$.

The likelihood analysis was performed on 4 catalogs:

- 2MRS catalog [9], taking out sources closer than $1 \mathrm{Mpc}$, as selected in [8], which traces the nearby matter.

- Swift-BAT AGNs [10], with the same selection as in [8], which includes both radio loud and quiet AGNs.

- $\gamma-\mathrm{AGN}$, this time selecting them from the 3FHL catalog [11] recently published by the Fermi collaboration. In [8] the previous 2FHL catalog was used. The selection applied here is the same, with the difference that, since the two catalogs are selected in different energy range, the reference flux used for weighting sources is defined as the integral from $10 \mathrm{GeV}$ to $1 \mathrm{TeV}$, rather than in the $50 \mathrm{GeV}-1 \mathrm{TeV}$ range as before.

- A sample of starburst galaxies selected based on their continuum emission at $1.4 \mathrm{GHz}$, used as a proxy of their UHECR flux. This selection has been updated by merging the data from [12] and [13] alredy reported in [8], with the addition of the Circinus Galaxy and sources selected with HEASARC Radio Master Catalog. ${ }^{3}$ The number of sources selected this way is 32 .

For further details on the catalog selection, please refer to the appropriate section in [8].

The best-fit TS obtained for each catalog at each energy threshold are shown in figure 3, left for starburst and $\gamma-$ AGN and right for Swift-BAT and 2MRS. It is possible to see that the maximum likelihood-ratio is found with starburst galaxies for $E>38 \mathrm{EeV}$, where a test statistics of 29.5 is obtained. At this energy threshold, the best-fit parameters are $f_{\text {aniso }}=11_{-4}^{+5} \%$ and $\theta=15_{-4}^{+5 \circ}$. This corresponds to a local p-value, accounting for the two fit parameters, of $4 \times 10^{-7}$, which has then to be penalized for the energy scan, obtaining a post-trial significance of $4.5 \sigma$. This is an increase of significance with respect to the results presented in the latest publication of the Collaboration

\footnotetext{
${ }^{3}$ https://heasarc.nasa.gov/W3Browse/master-catalog/radio.html
} 

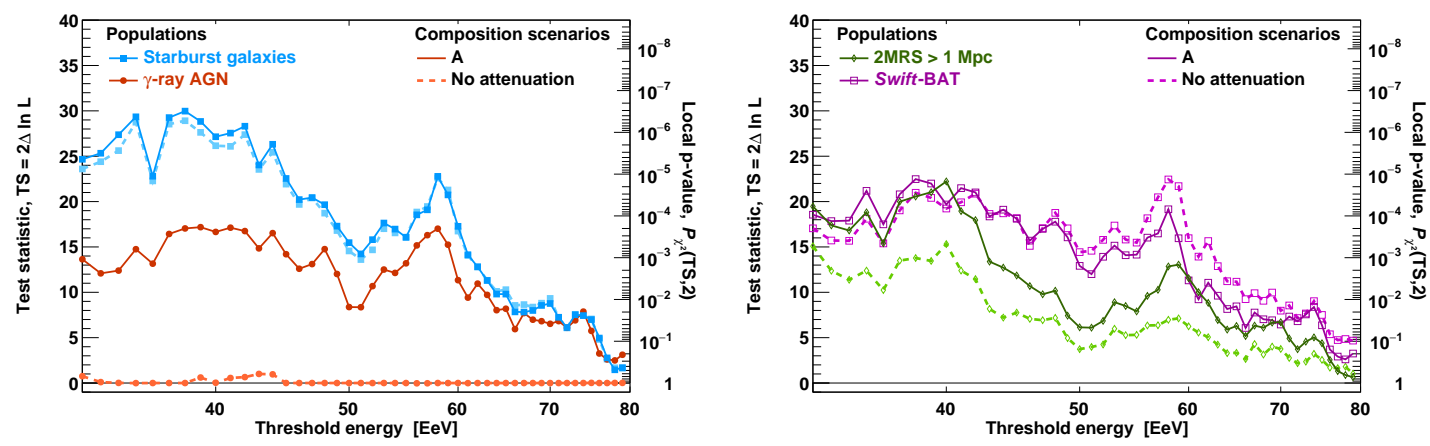

Figure 3: Maximum likelihood-ratio as a function of energy threshold for the models based on starburst galaxies and $\gamma$-AGN (left) and Swift-BAT and 2MRS (right). The dashed lines are the same obtained in the no-attenuation scenario.

\begin{tabular}{c||c|c|c|c|c|c} 
Catalog & $E_{\text {th }}$ & TS & Local p-value & post-trial & $f_{\text {aniso }}$ & $\theta$ \\
\hline Starburst & $38 \mathrm{EeV}$ & 29.5 & $4 \times 10^{-7}$ & $4.5 \sigma$ & $11_{-4}^{+5} \%$ & $15_{-4}^{+5} \circ$ \\
$\gamma-$ AGN & $39 \mathrm{EeV}$ & 17.8 & $1 \times 10^{-4}$ & $3.1 \sigma$ & $6_{-3}^{+4} \%$ & $14_{-4}^{+6} \circ$ \\
Swift-BAT & $38 \mathrm{EeV}$ & 22.2 & $2 \times 10^{-5}$ & $3.7 \sigma$ & $8_{-3}^{+4} \%$ & $15_{-4}^{+6} \circ$ \\
2MRS & $40 \mathrm{EeV}$ & 22.0 & $2 \times 10^{-5}$ & $3.7 \sigma$ & $19_{-7}^{+10} \%$ & $15_{-4}^{+7} \circ$ \\
\hline
\end{tabular}

Table 1: Values of the parameters that maximize the likelihood-ratio test against isotropy for the four different models as described in the text.

[8], where it was $4 \sigma .{ }^{4}$ A summary of the best-fit parameters obtained for all the four catalogs is reported in table 1 .

\section{Discussions and conclusion}

In this work, we have reported the updated results of searches for anisotropies in the highest energy cosmic rays. All the methods were used in previous analyses and were not changed for this update. With new data up to the end of August 2018, the excess around Centaurus A has increased as well as the significance of rejecting the isotropic hypothesis from a comparison with a starburst galaxies model, reaching $3.9 \sigma$ and $4.5 \sigma$ respectively.

Both the blind, full-sky search for excess and that centered on Centaurus A result in a maximum signal obtained at comparable energy thresholds (38 and $37 \mathrm{EeV}$ respectively) and similar top-hat angular scale ( $\psi=27^{\circ}$ and $28^{\circ}$ ). Similarly, the catalog-based analysis identifies the largest deviations form isotropy above $38-40 \mathrm{EeV}$ on an angular scale $\theta \sim 15 \pm 5^{\circ}$. The latter angular spread derives from a Fisher-Von Mises distribution, the equivalent of a Gaussian on the sphere, and would correspond to a top-hat scale $\psi \sim 1.59 \times \theta=24 \pm 8^{\circ} .5$

We note that the region with the most significant excess flux is densely populated with different types of nearby extragalactic objects. In particular, its center is $2^{\circ}$ away from the direction of Cen

\footnotetext{
${ }^{4}$ Note that in that case the energy scan ranged from 20 to $80 \mathrm{EeV}$.

${ }^{5}$ The scale factor 1.59 , close to the factor $\sqrt{3}$ expected from the scaling of the variances, corresponds to an optimal top-hat window, as used for instance in [14] and [15].
} 

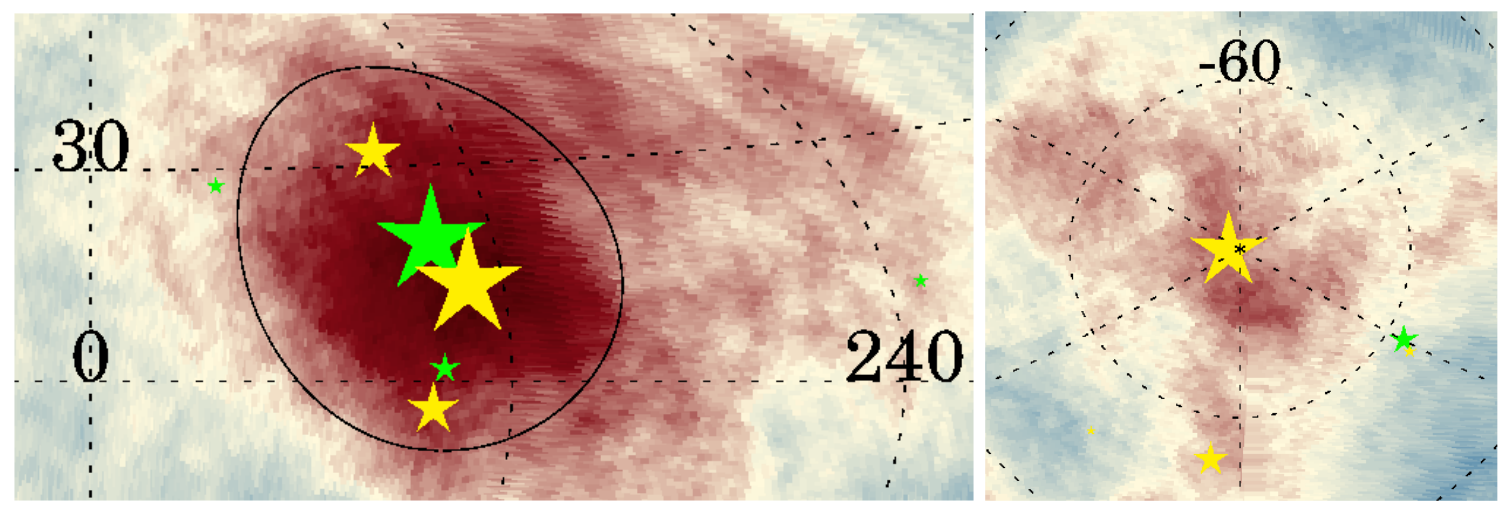

Figure 4: Zoom of the map in Figure 1 with the candidate sources that give the largest contribution to the expected flux in the best-fit models based on $\gamma$-AGNs (green) and starburst galaxies (yellow) superimposed. The area of the stars is proportional to their contribution. The left panel displays a black circle with radius $27^{\circ}$ centered at the position of the most significant flux excess, with also Cen A (the largest green star) and NGC 4945 (largest yellow star) visible. Right: the south Galactic pole excess, with NGC 253 visible as the largest yellow star.

A, which is the object with the largest contribution in the models based on $\gamma$-AGNs, Swift-BAT and 2MRS-galaxies, and $6^{\circ}$ away from the direction to NGC 4945, which is the object with the largest contribution in the model based on starburst galaxies. The best-fit flux-weighted models derived from the maximum-likelihood analysis predict that the fraction of CRs inside a circle with radius $27^{\circ}$ centred at R.A. $=202^{\circ}, \delta=-45^{\circ}$ is $13 \%$ (Starburst galaxies and Swift-BAT), $12.2 \%$ ( $\gamma$-AGNs) and $11.5 \%$ (2MRS) compared to $14.4 \%$ observed in data and $9.5 \%$ expected if the flux were isotropic. A more detailed view of this region is shown in figure 4, left panel, in which the black circle has radius $27^{\circ}$ and is centered at the position of the most significant overdensity. Also shown are the candidate sources in the models based on $\gamma$-AGNs and starburst galaxies, displayed as stars with area proportional to their individual contributions to the expected flux. Note that the model based on starburst galaxies also predicts excess flux around the position of NGC 253 (the galaxy with the second largest contribution in this model) near the southern Galactic pole, in which some excess flux is also indicated in data, as can be seen in figure 1. A more detailed view of this region is shown in figure 4, right panel. As the model based on starburst galaxies, the 2MRS-based model predicts excess flux near the southern Galactic pole, but it does also in other regions in which no excess is found in data, and that partly explains the relatively smaller value of its maximum teststatistics. The models based on $\gamma$-AGNs and Swift-BAT do not predict a significant flux excess around the southern Galactic pole. To disentangle between these different scenarios, other types of analyses may be useful, such as the search for magnetically induced signatures also presented in these proceedings [16]. Note also that we do not model in this analysis deflections in intervening magnetic fields that could change the distribution of flux expected from the same source candidates, given the large uncertainties that this modeling would involve.

We presented here the most significant result in a test for anisotropy in the arrival directions of UHECRs. We note that there is no rigorous way of penalizing these results for the numerous analyses performed within and outside the Pierre Auger Observatory Collaboration, in particular since many of such tests were performed using catalogs of astrophysical sources that show similar 
flux patterns in the sky. We will keep monitoring these interesting excesses, also with the additional information that is coming in the next few years with the observatory upgrade, Auger Prime [17].

\section{References}

[1] The Pierre Auger Collaboration, Science 357 (2017) 1266-1270

[2] V. Verzi for The Pierre Auger Collaboration. These proceedings. POS ( ICRC2019) 450

[3] The Pierre Auger Collaboration, Nucl. Instrum. Meth. A 798 (2015) 172.

[4] V. Verzi for The Pierre Auger Collaboration. Proceedings for the 33rd International Cosmic Ray Conference (ICRC 2013) arXiv:1307.5059

[5] E. Roulet for The Pierre Auger Collaboration, These proceedings. PoS ( ICRC2 019 ) 408

[6] The Pierre Auger Collaboration, Astrophys. J 804 (2015) 15

[7] U. Giaccari for The Pierre Auger Collaboration, PoS ( ICRC2 017 ) 483

[8] The Pierre Auger Collaboration, Astrophys. J. Lett. 853 (2018) L29

[9] J. P. Huchra, L. M. Macri, K. L. Masters, et al. Astrophys. J. Supp. 199 (2012) 26

[10] W. H. Baumgartner et al., Astrophys. J. Supp. 207 (2013) 19

[11] M. Ajello, et al. Astrophys. J. Supp. 232.2 (2017) 18

[12] M. Ackermann, M. Ajello,A. Allafort, et al. , Astrophys. J. 755 (2012) 164

[13] Becker, J. K., Biermann, P. L., Dreyer, J, Kneiske, T. M. 2009, ArXiv e-prints, arXiv:0901.1775

[14] The Pierre Auger Collaboration, Astroparticle Physics 27 (2007) 244

[15] The Pierre Auger Collaboration, Astrophys. J. , 760 (2012) 148

[16] M. Wirtz for The Pierre Auger Collaboration, these proceedings PoS (ICRC2019) 469

[17] The Pierre Auger Collaboration, arXiv:1604.03637 